\title{
Solving Bateman Equation for Xenon Transient Analysis Using Numerical Methods
}

\author{
Zechuan Ding \\ ${ }^{1}$ Illume Research, 4205 Xintianshiji Business Center, 125 Shixia Road, Shenzhen, China
}

\begin{abstract}
After a nuclear reactor is shutdown, xenon-135, an isotope with a very high thermal neutron absorption cross-section, will build up and reduce the reactivity considerably for a while. This is known as poisoning. However, the concentration xenon-135 would gradually decrease through decaying or absorbing neutron, making it necessary to suppress the reactivity in order to prevent the reactor to go critical or supercritical. Thus, it is important to predict the relationship between xenon poisoning and time after the reactor is shutdown to ensure the safety of the reactor. This paper reports the research on the prediction of xenon poisoning in a hypothetical nuclear reactor after it is shut down. In order to make the prediction, the Bateman equations of xenon (Xe) and iodine (I), which is of the form of an Ordinary Differential Equation (ODE) system, need to be solved. Two different methods, the fourth-order Runge-Kutta method and the matrix exponential method, were applied to solve the ODE system with MATLAB codes. The accuracies and computational efficiencies of the two method is also studied and compared.
\end{abstract}

\section{Introduction}

Ensuring safety is one of the most important goals, if not the most important goal, in today's nuclear energy industry. In most of the operating fleet of reactors, the nuclear fuel, in particular uranium-235, absorbs the thermal neutrons to maintain chain reaction to provide fission energy. However, there are many other isotopes present in the core that compete with uranium-235 for thermal neutrons, of which the isotopes with notably large cross-sections are referred to as reactivity poisons. Poisons can affect the reactivity management in many ways. Some poison materials are used in reactors for reactivity control or fuel management, whereas some fission products poisons, for example, can cause trouble to reactivity control and inherent safety of reactors.

Among all the fission products, xenon-135 has the most substantial effect on the reactivity due to its large absorption cross-section (one group absorption cross-section of 2.75 Mbarns in a standard PWR) [1]. This phenomenon is called xenon poisoning. After the shutdown of the reactor, the flux of thermal neutron will drop to almost zero. Consequently, the Xe-135 stops being produced from fission. Therefore, it is only produced through the decay of another nuclide iodine-135. However, similarly, Xe-135 does not vanish due to the absorption of thermal neutron, and it only vanishes through its $\beta$ decay. Because Xe-135 has a longer half-life (9.2 hours) than I-135 (6.7 hours), xenon decays at a lower rate than iodine does. As a result, a large amount of Xe-135 will build up in the reactor, and it absorbs a significant number of neutrons. The population of xenon will keep increasing until it reaches a peak and then decline. During this process, the reactivity of the reactor will drop at first and rise again, forming a period of time which the reactor cannot be restarted due to the very low reactivity, called the iodine pit.

More importantly, the decay of xenon after the xenon peak shutdown could release large amount of reactivity into the core. Even after reactor shutdown and the control rods are fully inserted, if the reactivity increase from the decay of xenon is too high, the reactor may reach criticality or supercriticality, threatening the safety of the reactor. When designing a reactor, the control rod system must be designed such that the shutdown margin (the reactivity difference between the core at hot full power and the core at cold zero power when xenon is fully decayed after shutdown, while the control rod with the largest worth is stuck out of the core) is maintained sufficiently positive at all times. Therefore, the precise prediction of the xenon poisoning is crucial in reactor operation and the design of new reactors. Conventional tools used to model xenon transient behaviours include stochastic or deterministic lattice physics codes such as Serpent or WIMS or nodal diffusion codes such as DYN3D and Panther. These codes perform flux calculation for each time step of the transient process and relatively accurately predicts spatial xenon distribution at each step, therefore predicting its transient behavior. This paper proposes to simplify the process of xenon transient calculation by numerically solve the one group Bateman equation using MATLAB for the iodine (I) - xenon (Xe) decay chain using homogeneous reactor properties. By doing so, quicker and reasonably accurate results can be 
achieved which will be beneficial for preliminary reactor designs studies.

This paper starts by introducing the Bateman equation, which describes the rate of the population change of a nuclide in a reactor. The Bateman equations for different isotopes form of a system of ordinary differential equations. This study tries to solve the system of Bateman equations governing the iodine-xenon decay chain to calculate the population change of nuclide xenon-135 after reactor shutdown, which could be used to calculate the xenon population. Two methods, the fourth-order Runge-Kutta (RK4) method and the matrix exponential method, are applied to solve the equation through codes. The performances of both methods are studied in terms of accuracy and efficiency. The fourth order Runge-Kutta method is found better in terms of calculation speed; however, the matrix exponential generates results with much higher accuracy. The two methods are then used to produce 3D plot of the "xenon poisoning surface" at different thermal neutron flux levels.

\section{Bateman equation for I-Xe decay chain}

Bateman equation is a mathematical model that describes the abundance of a nuclide in the decay chain of isotopes. In order to calculate the population of a particular isotope, one would solve the Bateman equation governing the decay chain that includes the very isotope of interest. The following is the decay chain of the fission product tellurium-135 that involves the production and decay of Xe-135 [1]:

$$
{ }_{52}^{135} \mathrm{Te} \rightarrow{ }_{53}^{135} \mathrm{I} \rightarrow{ }_{54}^{135} \mathrm{Xe} \rightarrow{ }_{55}^{135} \mathrm{Cs} \rightarrow{ }_{56}^{135} \mathrm{Ba}
$$

Let $I(t)$ and $X(t)$ be the population of iodine-135 and xenon-135 in the reactor. The equations for the I-135 and Xe-135 populations in a reactor, I and X, can then be written as:

$$
\begin{aligned}
& \frac{d}{d t} I(t)=\lambda_{T} T(t)-\lambda_{I} I(t) \#(1) \\
& \frac{d}{d t} X(t)=\lambda_{I} I(t)-\lambda_{X} X(t) \#(2)
\end{aligned}
$$

where $\lambda_{T}$ is the decay constant for tellurium-135,

$\lambda_{I}$ is the decay constant for I-135, and

$\lambda_{X}$ is the decay constant for Xe- 135 .

The half-life of tellurium (Te) is very short, which is roughly 11 seconds [1]. As a result, it can be assumed that I-135 is the primary fission product. Therefore, the first decay term, $\lambda_{T} T$ is ignored in equation (1).

Since the primary source of iodine is the fission, the rate of fission production of iodine is introduced to the equation. The equation can be then written as:

$$
\frac{d}{d t} I(t)=\gamma_{I} \Sigma_{f} \phi-\lambda_{I} I(t) \#(3)
$$

where $\gamma_{I}$ is the fission yield of I-135, and $\Sigma_{f} \phi$ is the fission rate.
The microscopic absorption cross-section of I-135 is so small that the loss rate of I-135 through absorbing neutron is very low compared to the decay rate and therefore can be ignored [1].

Besides the decay of iodine-135, fission can also contribute to the production of $\mathrm{Xe}-135$. Therefore, the fission rate of $\mathrm{Xe}-135$ is introduced to the Bateman equation. Besides the decay of Xe-135, the absorption of thermal neutron can also lead to the loss of Xe-135. Therefore, the full expansion of Bateman equation of xenon is obtained, which can be written as [1]:

$$
\frac{d}{d t} X(t)=\gamma_{X} \Sigma_{f} \phi+\lambda_{I} I(t)-\lambda_{X} X(t)-\sigma_{a X} X(t) \phi \#(4)
$$

where $\gamma_{X}$ is the fission yield of Xe-135,

$\sigma_{a X}$ is the microscopic absorption cross-section of $\mathrm{Xe}-135$, and

$\phi$ is the neutron flux.

The Bateman equations of iodine and xenon in equation (3) and (4) form a system of initial value ordinary differential equations. By solving the ODE system, the population of xenon and iodine after the shutdown of the reactor can be obtained as a function of time. When the reactor is shut down, the neutron flux $\phi$ is assumed to be zero instantaneously. Let $I_{0}$ and $X_{0}$ be the population of iodine and xenon at the time of shutdown. For the reactors that have been working long enough to reach equilibrium, $I_{0}$ and $X_{0}$ can be calculated through the equations since the rate of change of the iodine and xenon populations, $\frac{d}{d t} I(t)$ and $\frac{d}{d t} X(t)$ are all zero.

By applying integrating factor technique, representations of the population of iodine and xenon can be obtained:

$$
\begin{gathered}
I(t)=\frac{\gamma_{I} \Sigma_{f} \phi}{\lambda_{I}} e^{-\lambda_{I} t} \#(5) \\
X(t)=\Sigma_{f} \phi\left[\frac{\left(\gamma_{I}+\gamma_{X}\right)}{\lambda_{X}+\sigma_{a X} \phi} e^{-\lambda_{X} t}+\frac{\gamma_{I}}{\lambda_{I}-\lambda_{X}}\left(e^{-\lambda_{X} t}-e^{-\lambda_{I} t}\right)\right] \#(6)
\end{gathered}
$$

\section{Methods to solve Bateman equation}

\subsection{Runge-Kutta method}

The Bateman equations of iodine and xenon form a system of initial value ordinary differential equations. Many methods can be used to solve the initial-value ordinary differential equations with various orders, such as Euler method, midpoint method, etc. In this paper, the Fourth-order Runge-Kutta method and the matrix exponential method were selected to numerically solve the Bateman equation.

The Runge-Kutta method was developed by C. Runge and M. W. Kutta. It uses combinations of explicit and implicit iterative methods in temporal discretization to approximate solutions of ordinary differential equations. The well know Euler Method is also viewed as one of Runge-Kutta schemes [2].

Consider a general initial value ODE: 


$$
y^{\prime}=f(t, y), \quad y\left(t_{0}\right)=y_{0} \#(7)
$$

In the equation, $y$ is a function of time $t$, and the derivative of $y$ is a function of $t$ and $y$. The value of $y$ is $y_{0}$ at the initial time $t_{0}$.

The general form of each step in the solution of the fourth-order Runge-Kutta method is [3]:

$$
y_{n+1}=y+\frac{1}{6}\left(\Delta y_{1}+2 \Delta y_{2}+2 \Delta y_{3}+\Delta y_{4}\right) \#(8)
$$

In which

$$
\begin{gathered}
\Delta y_{1}=h f\left(t_{n}, y_{n}\right) \#(9) \\
\Delta y_{2}=h f\left(t_{n}+\frac{h}{2}, y_{n}+\frac{\Delta y_{1}}{2}\right) \#(10) \\
\Delta y_{3}=h f\left(t_{n}+\frac{h}{2}, y_{n}+\frac{\Delta y_{2}}{2}\right) \#(11) \\
\Delta y_{4}=h f\left(t_{n}+h, y_{n}+\Delta y_{3}\right) \#(12)
\end{gathered}
$$

Here $y_{n+1}$ is the RK4 approximation of $y\left(t_{n+1}\right)$. $y_{n+1}$ is obtained by the adding the weighted average of four increments to $y_{n}$. These increments are represented by equation (9) to (12). They are the product of the numerical interval $\mathrm{h}$ between $t_{n+1}$ and $t_{n}$, and an estimated slope specified by function $\mathrm{f}$ on the right-hand side of the differential equation $f(t, y)$.

The specific steps of solution of the Bateman equation by the fourth-order Runge-Kutta method is:

$$
\begin{gathered}
I_{n+1}=I_{n}+\frac{1}{6}\left(\Delta I_{1}+2 \Delta I_{2}+2 \Delta I_{3}+\Delta I_{4}\right) \#(13) \\
X_{n+1}=X_{n}+\frac{1}{6}\left(\Delta X_{1}+2 \Delta X_{2}+2 \Delta X_{3}+\Delta X_{4}\right) \#(14)
\end{gathered}
$$

In which

$$
\begin{gathered}
\Delta I_{1}=\Delta t\left(\gamma_{I} \Sigma_{f} \phi-\lambda_{I} I_{0}\right) \#(15) \\
\Delta X_{1}=\Delta t\left[\gamma_{X} \Sigma_{f} \phi+\lambda_{I} I_{0}-\left(\lambda_{X}+\sigma_{a X} \phi\right) X_{0}\right] \#(16) \\
\Delta I_{2}=\Delta t\left[\gamma_{I} \Sigma_{f} \phi-\lambda_{I}\left(I_{0}+\frac{1}{2} \Delta I_{1}\right)\right] \#(17)
\end{gathered}
$$$$
\Delta X_{2}=\Delta t\left[\gamma_{X} \Sigma_{f} \phi+\lambda_{I}\left(I_{0}+\frac{1}{2} \Delta I_{1}\right)-\left(\lambda_{X}+\sigma_{a X} \phi\right)\left(X_{0}+\frac{1}{2} \Delta X_{1}\right)\right] \#(18)
$$$$
\Delta I_{3}=\Delta t\left[\gamma_{I} \Sigma_{f} \phi-\lambda_{I}\left(I_{0}+\frac{1}{2} \Delta I_{2}\right)\right] \#(19)
$$$$
\Delta X_{3}=\Delta t\left[\gamma_{X} \Sigma_{f} \phi+\lambda_{I}\left(I_{0}+\frac{1}{2} \Delta I_{2}\right)-\left(\lambda_{X}+\sigma_{a X} \phi\right)\left(X_{0}+\frac{1}{2} \Delta X_{2}\right)\right] \#(20)
$$$$
\Delta I_{4}=\Delta t\left[\gamma_{I} \Sigma_{f} \phi-\lambda_{I}\left(I_{0}+\Delta I_{3}\right)\right] \#(21)
$$$$
\Delta X_{4}=\Delta t\left[\gamma_{X} \Sigma_{f} \phi+\lambda_{I}\left(I_{0}+\Delta I_{3}\right)-\left(\lambda_{X}+\sigma_{a X} \phi\right)\left(X_{0}+\Delta X_{3}\right)\right] \#(22)
$$

Where $\Delta t$ is the time interval, $I_{0}$ is the initial value of iodine, and $X_{0}$ is the initial value of xenon.
The advantage of Runge-Kutta is that it applies the single-step method and has a high precision, and it is easy to change the step length when doing the calculation. The disadvantage is that it has to calculate the function value $\mathrm{f}$ for four times for each increment. Nevertheless, the amount of calculation is not a problem today due to the advanced computer technology. Therefore, the fourth-order Runge-Kutta method is one of the common methods that solve the differential equations.

The Fourth-order Runge-Kutta method is implemented in MATLAB to solve the Bateman equations for the iodine-xenon decay chain. Appendix A illustrates the algorithm employed in the MATLAB script to perform the calculation for xenon population transient of 70 time steps 3 otalling 70 hours after reactor shutdown.

\subsection{Matrix exponential method}

In mathematics, the matrix exponential is a matrix function on square matrices analogous to the ordinary exponential function. It is used to solve systems of linear differential equations. MATLAB uses the Padé approximant to compute the matrix exponential [4]. It is applicable to solve the Bateman equations.

The general form of initial value problem of an ODE system is:

$$
\frac{d \vec{x}}{d t}=A \vec{x}, \quad \vec{x}\left(t_{0}\right)=\vec{x}_{0} \#(23)
$$

In the equation, vector matrix $\vec{x}$ is a matrix that includes functions of time $t$, and the derivative of $\vec{x}$ equals to the product of $\vec{x}$ and a constant coefficient matrix A. The value of $\overrightarrow{\mathrm{x}}$ is $\overrightarrow{\mathrm{x}}_{0}$ at the initial time $t_{0}$.

The solution to the equation (23) is:

$$
\vec{x}(t)=e^{\left(t-t_{0}\right) A} \vec{x}_{0} \#(24)
$$

The vector matrix that need to be solved contains the functions of time with respect to the population of xenon and the population of iodine. Then, the constant coefficient matrix is calculated, after which the Bateman equation can be solved.

The solution of the initial value problem of the system of Bateman equation in equation (3) and (4) is:

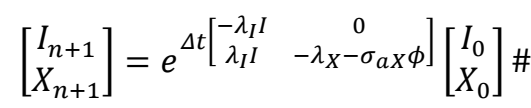

The matrix exponential method as described by equation (25) is also implemented in MATLAB to solve the Bateman equation for the iodine-xenon decay chain to predict xenon population under reactor shutdown transient. The algorithm listed in Appendix A is also used to implement the matrix exponential to simulate xenon transient behaviour after reactor shutdown for 70 hours.

\section{Benchmark study}


Once implemented in MATLAB, the two methods, Runge-Kutta and the matrix exponential, are used to perform a benchmark test for a hypothetical reactor using realistic reactor parameter inputs. These parameters are tabulated in Table 1. The reactor is assumed to be homogenous and non-leaking, as assumed also in Section 2. The cross-section used are all one-group values.

Table 1. Reactor parameters: independent variables used in Bateman equation.

\begin{tabular}{|c|c|c|}
\hline$\gamma_{I}$ & Fission yield of I-135 & 0.061 \\
\hline$\gamma_{X}$ & Fission yield of Xe-135 & 0.003 \\
\hline$\lambda_{I}$ & $\begin{array}{c}\text { Decay constant of I-135 } \\
\left(\mathrm{s}^{-1}\right)\end{array}$ & $2.874 \times 10^{-5}$ \\
\hline$\lambda_{X}$ & $\begin{array}{c}\text { Decay constant of Xe-135 } \\
\left(\mathrm{s}^{-1}\right)\end{array}$ & $2.027 \times 10^{-5}$ \\
\hline$\sigma_{a X}$ & $\begin{array}{c}\text { Microscopic absorption } \\
\text { cross-section of Xe-135 } \\
\left(\mathrm{cm}^{2}\right)\end{array}$ & $2.75 \times 10^{-18}$ \\
\hline$v$ & $\begin{array}{c}\text { \# of neutrons released per } \\
\text { fission }\end{array}$ & 2.3 \\
\hline$\phi$ & $\begin{array}{c}\text { Thermal neutron flux } \\
\left(\mathrm{cm}^{-2} \mathrm{~s}^{-1}\right)\end{array}$ & $4.42 \times 10^{20}$ \\
\hline$\Sigma_{f}$ & $\begin{array}{c}\text { Macroscopic absorption } \\
\text { cross-section }\left(\mathrm{cm}^{2}\right)\end{array}$ & 0.008 \\
\hline \multicolumn{2}{|c|}{} \\
\hline
\end{tabular}

In both methods, the function of poisoning in 70 hours after shutdown is investigated, so the total length is 252000 seconds, which is divided into 70 steps with a step length of 3600 seconds. In the hypothetical reactor being investigating, the thermal neutron flux is equal to $4.42 \times 10^{20}$ neutrons per 4entimetre squared per second, and macroscopic absorption cross-section is equal to $0.008 \mathrm{~cm}^{2}$.

By applying the two methods, the codes can investigate the xenon and iodine population. Then, the codes are able to calculate the poisoning. The function of poisoning used in the code is represented as:

$$
\rho_{X e}=-\frac{\sigma_{a X} X}{v \Sigma_{f}} \#(26)
$$

The xenon poisoning level with respect to time after a reactor shutdown from different power levels simulated using the RK4 method is plotted in Figure 1. It is assumed that at $t=0$ hour, the reactor (i.e. xenon population) is at equilibrium at the specified power level. The graph of xenon poisoning with respect to time is a curve that peaks very rapidly at first and then decays at a smaller slope. The graph show that the poisoning reaches its peak at around 11 hours after the shutdown and began to drop. It is also noticed that the higher the power level is before the reactor shutdown, the higher the peak of the xenon poisoning after shutdown.

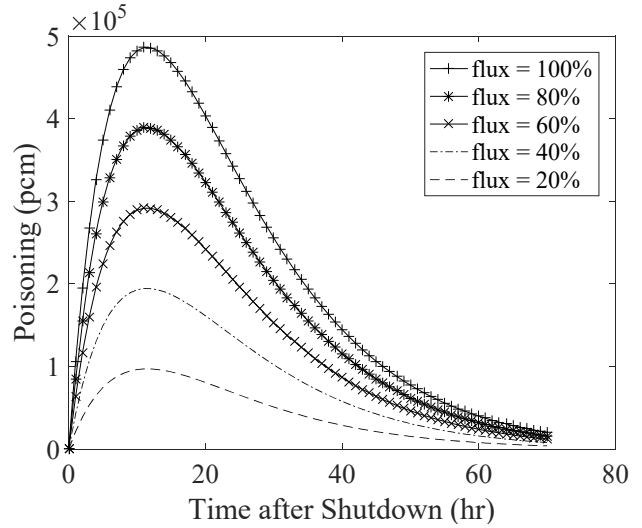

Fig. 1. Xenon Poisoning transient at different flux levels using the RK4 method.

The xenon poisoning transient result calculated using the matrix exponential method implemented in MATLAB. The two graphs show very similar pattern is plotted in Figure 2.

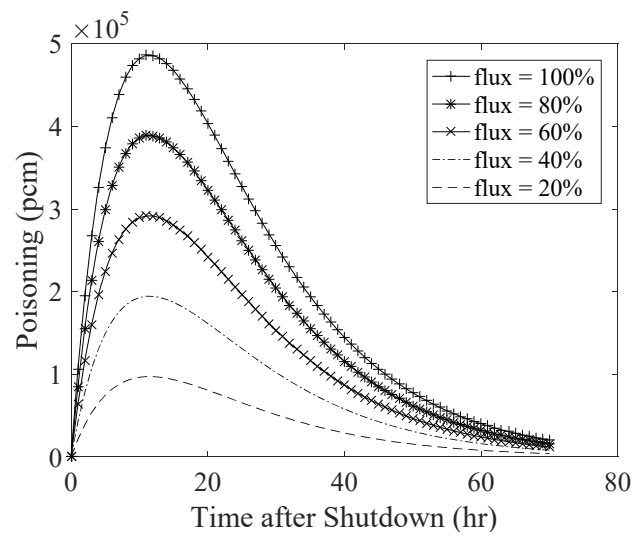

Fig. 2. Xenon Poisoning transient at different flux levels using the Matrix Exponential Method.

To compare the accuracy of the two solutions, the percentage error of each method with respect to analytical solution as presented in equation (6) is calculated. Figure 3 plots the percentage error of xenon poisoning of the full power case with respect to time after reactor shutdown using the RK4 method and Figure 4 plots the percentage error using the matrix exponential.

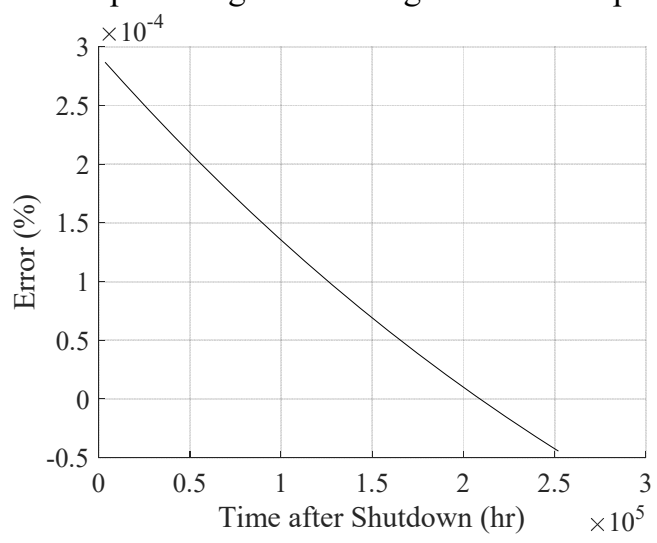

Fig. 3. Percentage Error of the RK4 Method. 


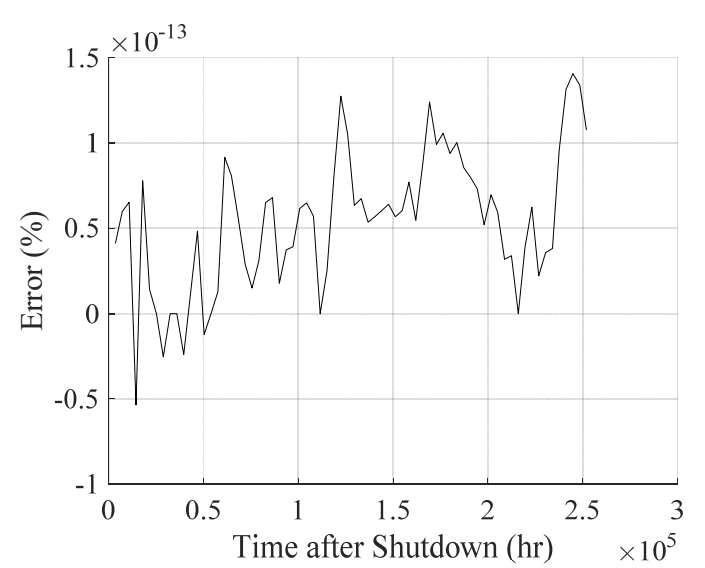

Fig. 4. Percentage Error of the Matrix Exponential Method.

As shown on the graphs above, the error of result from the RK method stays within $0.0003 \%$. For the result generated by the matrix exponential method, the error stays within $1.5 \times 10^{13} \%$. Clearly, the matrix exponential gives more accurate solutions.

The calculation time of each method is also tested to investigate the trade-off between accuracy and computational expense of the two methods. The average operation time of 300 runs is around 0.09 milliseconds using the RK4 method and is around 5 milliseconds when using the matrix exponential method.

Both methods are accurate and can provide good approximation of the "true" analytical answer. The matrix exponential method requires longer calculation time, but it is much more accurate than the Fourth-order Runge-Kutta method. The problem investigated at this stage of the project is relatively simple and both methods can provide accurate results within a very short time frame, therefore the matrix exponential is the preferred method. However, when the problem becomes more complicated, computational efficiency might become a much greater concern. The less computationally expensive method RK4 could become the appropriate.

\section{Results and discussion}

After the two models have been successfully benchmarked against the analytical solution, they are used to produce a xenon transient "surface" for different reactor operating conditions, i.e. neutron flux and one-group macroscopic fission cross-section. Figure 5 visualizes the $3 \mathrm{D}$ surface illustrating the shutdown transient of xenon poisoning reactor under different reactor equilibrium power levels before the shutdown. This essentially is Figure 1 presented in 3D space. In the calculation presented in Figure 1, a full power neutron flux of $4.42 \times 10^{20} / \mathrm{s} \cdot \mathrm{m}^{2}$ as the value of neutron flux. A range of flux levels from $80 \%$ to $120 \%$ of the full power flux level with a $4 \%$ increment is explored. The importance of this "xenon transient surface" is that once the governing equation of the surface is known, the xenon population can be predicted using the surface equation without needing to re-calculate Bateman equation. This will speed up the design safety check process.

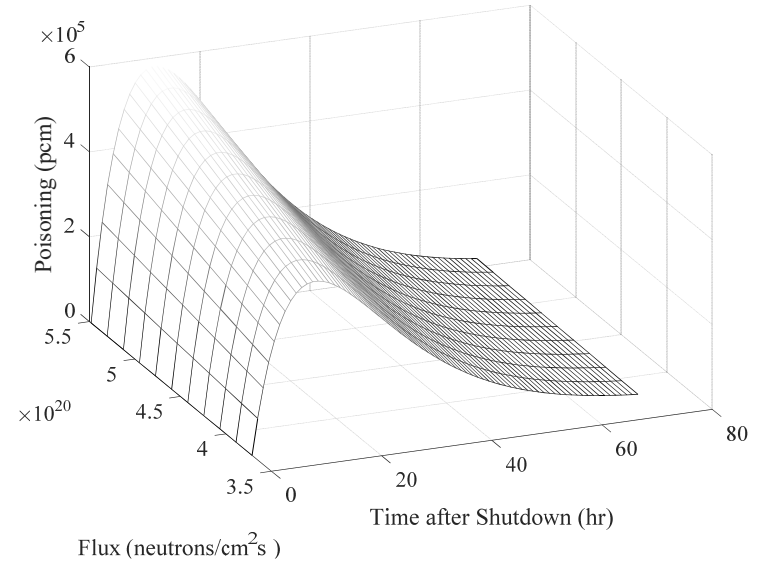

Fig. 5. Xenon Poisoning under Different Neutron Fluxes using the Matrix Exponential method.

Similar pattern can be seen in Figure 5 as seen in Figure 1 and 2. Xenon poisoning peaks around $11 \mathrm{hrs}$ after reactor shutdown and then decays with a relatively slower rate. The height of the peak depends on the homogeneous one group neutron flux, in other words, the reactor power before reactor shutdown. The greater the flux is, the higher the poisoning peak is. The height of the xenon peak reaches almost $5 \times 10^{5} \mathrm{pcm}$ at $100 \%$ power, meaning that a significant amount of reactivity is suppressed by the xenon poisoning. This means that there will be a time frame after which the reactor shutdown during which the reactor could not be restarted because of the lack of access reactivity. Even if the reactor restarts before the xenon reaches its peak, the continual production of xenon from iodine decay could still shut the reactor down. This is also one of the reasons why nuclear power plants normally operate as base load power provider, even during times of low electricity demand. Once the reactor shuts down, the core is theoretically impossible to restart until xenon poisoning decays to a level lower than the reactivity of the fuels, and the economic loss during the shutdown period can be significant.

Furthermore, power level fluctuations also lead to similar behaviours as discussed previously. When the core goes from a high flux state to a low flux state, xenon is also built up and supresses the core reactivity before reaching to a new equilibrium state at a lower poisoning level. During the xenon peak and decay, if the reactor were to be maintained critical, control rods would have to be gradually withdrawn to release more neutrons to the core during the xenon peak and then inserted again once xenon starts to decay. This makes reactivity control of the core extremely complicated and must be handled meticulously. The infamous Chernobyl accident was in fact caused by reactivity release of the decayed xenon poisoning [5]. 


\section{Appendix A}

Algorithm used to implement the RK4 and the matrix exponential methods in MATLAB.

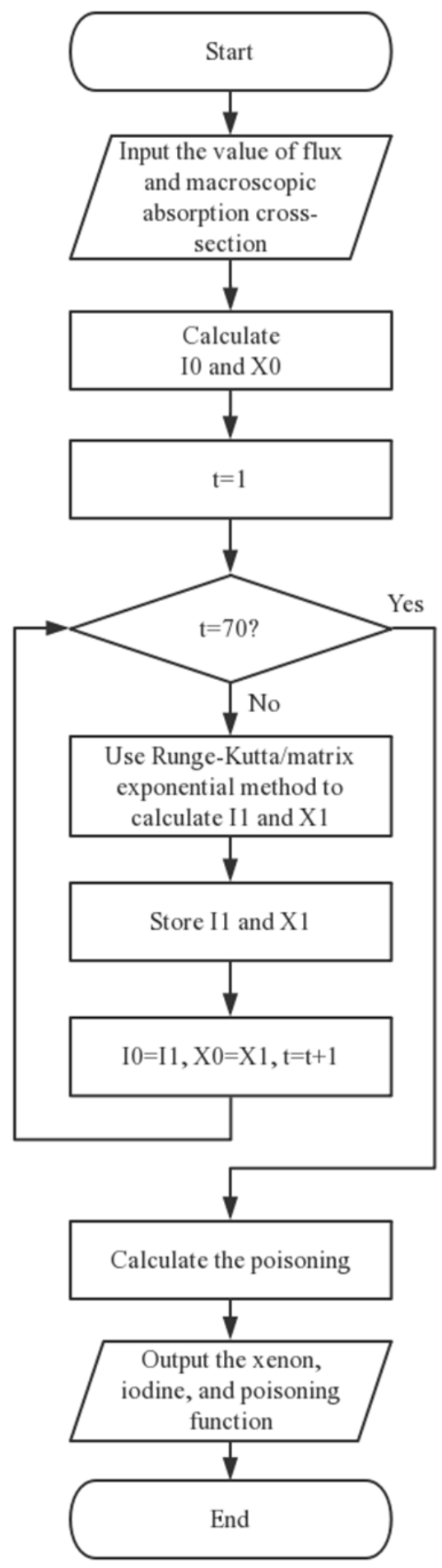

\section{Conclusion and future work}

Due to its extremely high absorption cross-section, xenon plays a vital role in reactor safety during operation; therefore, understanding xenon's transient behaviour under reactor power level change is of crucial significance for reactor design and operation. In this paper, two mathematical methods, i.e. Runge-Kutta and the matrix exponential, have been employed using MATLAB script to solve Bateman equations for the iodine-xenon decay chain to model xenon population behaviour under "reactor shutdown" transient scenarios. Results obtained from these two methods agree within an acceptable range with an error smaller than $3 \times 10^{-4} \%$. The models are then used to test how the transient behaviour of xenon changes with respect to neutron flux, i.e. reactor power level. It is seen that with higher flux, a higher xenon poisoning peak is observed, longer time required to decay. The xenon build-up could render the reactor core impossible for restart during some time frame after a shutdown. Xenon fluctuation also complicates the reactivity control during operation if the core power level changes.

It must be acknowledged that the methods developed in this paper, however, can only provide rough estimations of xenon transient as they currently only solve one-group Bateman equations in homogeneous reactor environment. There are many factors that have not been included in the models that could affect the calculation results, such as spatial variation of nuclide distribution and cross-sections and energy level dependence of the parameters. Implementing these factors would require more sophisticated methods such as nodal diffusion methods, Method of Characteristics or Monte-Carlo neutron transport methods; however, as explained earlier, these methods require longer calculation time, especially when doing transient analysis and therefore might not be the best fit for preliminary reactor design stage. The simple models developed in this project can be modified in future work to include multi-group calculation, spatial heterogeneity and couple to legacy codes to perform xenon transient analysis on the fly of reactor design with more detailed reactor specific information.

\section{References}

1. E.E. Lewis, Fundamentals of Nuclear Reactor Physics, Chapter 10, pp. 243-258, Academic Press (2008).

2. DEVRIES, Paul L., HASBUN, Javier E., A first course in computational physics, Second edition, pp. 215, Jones and Bartlett Publishers (2011).

3. Joe D. Hoffman, Numerical Methods for Engineers and Scientists, pp. 370-372, Marcel Dekker (2001).

4. Higham, N. J., The Scaling and Squaring Method for the Matrix Exponential Revisited, pp. 1179-1193, SIAM J. Matrix Anal. Appl., 26(4), (2005).

5. C.D. Bowman, What Happened at Chernobyl?, Science (New York, 2011). 